\title{
Editorial
}

Prof. Dr. med. Eckard Hamelmann, Direktor der Klinik für Kinder- und Jugendmedizin, Ruhr-Universität Bochum, Leiter der Sektion Pädiatrie der Deutschen Gesellschaft für Allergologie und klinische Immunologie (DGAKI)

\section{Update Allergie und Asthma}

A llergische Erkrankungen, Neurodermitis und Asthma sind längst zu Volkskrankheiten des 21. Jahrhunderts geworden. Besonders Kinder und Jugendliche sind in erheblichem Maße betroffen: Mindestens jedes vierte Kind wird innerhalb der ersten 18 Lebensjahre an der einen oder anderen Form des allergischen Formenkreises erkranken. Deswegen wird jeder Pädiater - vom ausgewiesenen Spezialisten bis zum breit aufgestellten Kinder- und Jugendarzt - immer häufiger und immer stärker mit den Problemen rund um diese „Sie sehen: Erkrankungen konfrontiert.

Das Thema Allergie und Asthma bleibt sicher

Es ist mir daher eine besehrspannend!" sondere Freude, Ihnen hiermit ein Themenheft vorlegen zu dürfen, welches den "state of the art" zur pädiatrischen Allergologie zusammenfasst. Ein engagiertes und besonders ausgewiesenes Autorenteam stellt den aktuellen Stand rund um die wichtigsten Fragen dar und gibt einen Ausblick auf die zukünftigen Entwicklungen. Die Übersichten zu den genetischen, epidemiologischen und immunologischen Grundlagen erlauben auch dem Nicht-Spezialisten einen raschen und fundierten Einblick in die komplexen Zusammenhänge der Ursachen und der Pathogenese von Allergie und Asthma. Die gängigen und evidenzbasierten Therapieoptionen für Neurodermitis, Nahrungsmittelallergie und Asthma inklusive des schweren Asthmas werden praxisnah vermittelt und kritisch kommentiert.
Einen besonderen Schwerpunkt legen wir auf das Thema der Prävention: Was kann man heute belasteten Eltern raten, damit eine Allergie bei ihren Kindern möglichst von vorneherein verhindert wird? Und wie können wir bei Nachweis einer allergischen Sensibilisierung schon im Kindesalter durch die spezifische Immuntherapie kausal in die Krankheitsentwicklung eingreifen? Schließlich wagen wir am Ende dieses Heftes einen Ausblick in die Zukunft und stellen die möglichen Therapieoptionen von morgen und übermorgen vor.

Sie sehen: Das Thema Allergie und Asthma bleibt sicher sehr spannend! Bis neue Therapieoptionen zur Verfügung stehen, sollten unbedingt die von den Fachgesellschaften veröffentlichten Leitlinien als Grundlage der aktuellen Therapie herangezogen werden. Dringend ist davor zu warnen, aus Frustration über schwere, rezidivierende oder chronische Verläufe zu Mitteln und Wegen zu greifen, die zwar augenfällig gut verkäuflich sind oder damit werben, ungefährlich und dabei auch noch hochwirksam zu sein. Bei näherer Betrachtung können diese jedoch fast nie das halten, was sie versprechen.

Viel Spaß beim Lesen wünscht Ihnen

Ihr

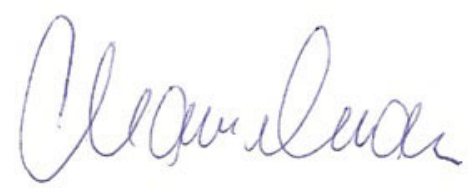

